\title{
Use of Initial Modified RECIST Tumor Response Evaluation Criteria for Predicting Survival in Patients with Hepatocellular Carcinoma Undergoing Transarterial Chemoembolization with Drug-Eluting Beads
}

\author{
Natália Sousa Freitas Queiroz ${ }^{1}$, Luciana Kikuchi' ${ }^{1}$, Regis Otaviano Franca Bezerra ${ }^{2}$, \\ Regiane S. S. M. Alencar ${ }^{1}$, Aline Lopes Chagas ${ }^{1}$, Cláudia Megumi Tani' ${ }^{1}$, \\ Márcio Augusto Diniz ${ }^{1}$, Aline Cristine Barbosa Santos ${ }^{2}$, Airton Mota Moreira², \\ Manoel de Souza Rocha' ${ }^{2}$ Luiz Augusto Carneiro D'Albuquerque1, \\ Francisco César Carnevale², Flair José Carrilho ${ }^{1}$ \\ ${ }^{1}$ São Paulo Clínicas Liver Cancer Group, Instituto do Câncer do Estado de São Paulo, Hospital das Clínicas, \\ Department of Gastroenterology, University of São Paulo School of Medicine, São Paulo, Brazil \\ ${ }^{2}$ São Paulo Clínicas Liver Cancer Group, Instituto do Câncer do Estado de São Paulo, Hospital das Clínicas, \\ Department of Radiology, University of São Paulo School of Medicine, São Paulo, Brazil \\ Email: lucianakikuchi@usp.br
}

Received 26 August 2015; accepted 29 November 2015; published 2 December 2015

Copyright (C) 2015 by authors and Scientific Research Publishing Inc.

This work is licensed under the Creative Commons Attribution International License (CC BY). http://creativecommons.org/licenses/by/4.0/

(c) (i) Open Access

\begin{abstract}
Introduction: Transarterial chemoembolization (TACE) reduces tumor growth and increases survival in patients with hepatocellular carcinoma (HCC). Drug-eluting beads (DEB) deliver slow-release chemotherapy and reduce systemic toxicity during TACE. This study correlated initial tumor response according to modified RECIST (mRECIST) criteria and 1-year survival in patients with HCC treated with TACE-DEB, and identified predictors of tumor response. Methods: Fifty-two patients with HCC received TACE-DEB loaded with doxorubicin 75 mg during a 6-month period. Tumor response was evaluated 1 month after the procedure according to mRECIST criteria. Results: Most patients were cirrhotic and etiology of liver disease was hepatitis C in 26/52 (50\%). Similar numbers of patients had Barcelona Clinic Liver Cancer (BCLC) A and BCLC B disease. Most patients had one nodule (66\%). Complete response (CR) was achieved in 12/52 (23\%), partial response in $19 / 52(37 \%)$, stable disease in $4 / 52(8 \%)$ and progressive disease in $17 / 52(32 \%)$. Largest HCC


$\leq 58 \mathrm{~mm}$ and BCLC stage A were associated with CR. The 1-year survival was $74 \%$, with survival rates of $95 \%$ and $56 \%$ in the BCLC $A$ and $B$ groups, respectively. Variables reflecting tumor extension were associated with better survival. CR according to mRECIST criteria was a predictor of better 1-year survival $(100 \%$ vs. $64 \%, P<0.05)$. Conclusion: BCLC $A$ and CR according to $\mathrm{mRECIST}$ criteria predict improved 1-year survival in patients with HCC treated with TACE-DEB. Further studies are needed to evaluate other predictors of survival and to determine if tumor response predicts long-term survival.

\section{Keywords}

Hepatocellular Carcinoma, mRECIST Criteria, Transarterial Chemoembolization, Overall Survival, Drug-Eluting Beads

\section{Introduction}

Hepatocellular carcinoma (HCC) is the fifth most common malignancy worldwide, with half a million new cases reported every year [1] [2]. Transarterial chemoembolization (TACE) is the current standard of care for patients with multifocal, large volume and non-resectable tumors confined to the liver, with good performance status and preserved liver function [3] [4]. TACE has also been advocated as a potential therapeutic option in patients at an earlier stage (i.e., single nodule or up to 3 nodules under $3 \mathrm{~cm}$ ), for whom liver resection, transplantation or ablation is not feasible [5]-[7].

TACE technique consists in the intra-arterial administration of a chemotherapeutic drug through the feeding hepatic artery of the tumor followed by embolization of the blood vessel, resulting in a cytotoxic effect combined with ischemia. Chemoembolization with drug-eluting beads (TACE-DEB) increases local concentrations of the drug with negligible systemic toxicity. DEBs are embolizing devices that slowly release the antineoplastic agent into the tumor. They have been associated with reduced systemic side effects and increased local levels of antineoplastic agents, compared with other modes of administration [8]-[11]. Chemoembolization has been used for several years, although techniques vary widely between centers, in terms of chemotherapeutic drugs (doxorubicin, mitomycin, cisplatin, and mixtures), embolic agents, doses, and schedules used. Although response rates vary, evidence suggests acceptable survival benefit, particularly at 3 years [12].

Many methods have been used to assess treatment efficacy. The response evaluation criteria in solid tumors (RECIST) are based on tumor shrinkage and are widely used in oncology to evaluate treatment response [1]. However, the validity of this method for assessing loco-regional treatment response has been challenged, especially in the case of TACE, which causes acute tumor devascularization and necrosis, which are not always correlated with a reduction in tumor size [2]. European Association for the Study of the Liver (EASL) and American Association for the Study of Liver Diseases (AASLD) guidelines recently adopted a modified version of the World Health Organization (WHO) criteria, mRECIST, which take account of changes in the degree of tumor arterial enhancement [5].

The aim of this study was to assess the correlation between initial tumor response according to mRECIST criteria and the 1-year survival rate in patients with HCC treated with TACE-DEB, and to identify predictors of tumor treatment response.

\section{Materials and Methods}

This study was designed as a single-center retrospective analysis and was carried out with pre-approval by the local institutional review board (Registration ID: 368/12; Instituto do Câncer do Estado de São Paulo (ICESP), Brazil) according to the ethical guidelines of the Declaration of Helsinki.

\subsection{Patient Population}

Fifty-two consecutive patients with HCC underwent 65 episodes of TACE-DEB loaded with doxorubicin $75 \mathrm{mg}$ between November 2010 and April 2011. The patient and tumor characteristics and the underlying liver diseases are summarized in Table 1. 
Table 1. Baseline characteristics.

\begin{tabular}{cc}
\hline Variable & N \\
\hline Age, yr $(\leq 65 />65)$ & $25 / 27$ \\
Gender (M/F) & $39 / 13$ \\
Child-Pugh (A/B) & $35 / 17$ \\
Etiology (HCV/HBV/alcohol/NASH) & $26 / 8 / 10 / 8$ \\
Number of nodules (1/>1) & $18 / 34$ \\
Largest HCC size, mm $(\leq 40 />40)$ & $27 / 25$ \\
Sum of HCCs, mm $(\leq 60 />60)$ & $38 / 14$ \\
AFP, ng/ml $(\leq 25 />25)$ & $26 / 26$ \\
BCLC $(\mathrm{A} / \mathrm{B})$ & $26 / 26$ \\
\hline
\end{tabular}

The inclusion criteria for TACE-DEB were: i) non-resectable HCC; ii) contraindication to ablation procedures; iii) liver-confined disease; iv) good performance status ( 0 - 1); v) adequate liver function (Child-Pugh A/B); vi) HCC diagnosis according to EASL/AASLD guidelines [6]. Patients on the liver-transplant list were not excluded.

During the study period, 52 eligible patients diagnosed with HCC were referred for chemoembolization treatment, 80 procedures were planned, but 15 procedures were contraindicated because of: i) arterioportal fistula (7 cases); ii) portal vein (1 case) or hepatic artery (1 case) thrombosis; iii) liver dysfunction (6 cases).

\subsection{Diagnosis}

HCC was diagnosed by contrast-enhanced computed tomography (CT) and magnetic resonance imaging (MRI), according to AASLD/EASL criteria [6]. CT was performed based on our exam protocol that included non-contrast, arterial, portal and equilibrium phase assessments. MRI exams included T2-weighted images, diffusion, in-phase, out-of-phase and pre- and post-contrast T1-weighted images. The image apparatus was available in the institution where the study was performed. CT (Philips Brillance CT 64-channel scanner) was carried out with iodine contrast (Ultravist ${ }^{\circledR}$, Bayer) and MRI (GE Healthcare MR Signa HDx 1.5T) with Gadolinium (Dotarem ${ }^{\circledR}$, Guerbet).

\subsection{Treatment Protocol}

The interventional procedure was performed via percutaneous femoral artery access using a $5 \mathrm{~F}$ introducer and a 5F diagnostic catheter. Liver tumor arterial supply was assessed by arteriograms to confirm the absence of arterio-portal fistulas, and to detect possible parasitic vascularization. A 2.3F coaxial microcatheter was advanced into the corresponding segmental artery and forwarded to subsegmental levels, according to tumor size and arterial blood supply. One vial of DC-Bead (Biocompatibles, UK), 300 - $500 \mu \mathrm{m}$, was loaded with $75 \mathrm{mg}$ of doxorubicin (Pfizer Pharma GmbH, Karlsruhe, Germany). The embolization endpoint was based on blood-flow stasis. In case of incomplete blood-flow stasis, additional bland embolization was considered, at the discretion of the investigator, using the same size of Bead Block (Biocompatibles, UK). TACE-DEB was performed every 2 3 months until one of the following endpoints was reached: i) complete tumor devascularization; ii) technically impossible to embolize the residual tumor; iii) contraindications to TACE; iv) total resection or ablation of tumor by subsequent surgery or local ablation.

Peri-interventional management included complete cardiopulmonary monitoring (electrocardiogram, blood pressure, oxygen saturation), and antiemetic prophylaxis (ondansetron $4 \mathrm{mg}$ intravenously (i.v.)). There was no antibiotic prophylaxis.

Post-embolization syndrome (e.g., abdominal pain, nausea, vomiting, and/or fever), was treated with ondansetron 4 - 16 mg i.v. (Hangzhou Pharm \& Chem Co., Ltd.), tramadol cloridrate 50 - 150 mg i.v. (Cristalia Produtos Químicos Farmacêuticos Ltd.) and dipyrone 500 - 4000 mg i.v. (Farmace Indústria Químico-Farmacêutica Cearense Ltd.). Clinical examinations were performed within $24 \mathrm{~h}$ after TACE and before discharge. 


\subsection{Follow-Up}

Radiological assessment of treatment response was carried out using CT or MRI 1 month after the procedure, according to mRECIST criteria [5]. Images were reviewed by a radiologist with 8 years expertise in abdominal imaging. Tumor treatment response was defined as complete response (CR), based on the disappearance of any tumoral arterial enhancement in all target lesions, or partial response (PR) defined as a 30\% decrease in the sum of the viable target lesions. Disease progression (PD) was defined as a $20 \%$ increase from baseline, and stable disease (SD) was defined as any case that did not meet the criteria for PR or PD.

Patients were followed up every 3 months until death or loss to follow-up in the outpatient setting.

\subsection{Statistical Analysis}

The results were presented as mean \pm standard deviation or median and range. Survival was assessed considering the time between TACE-DEB and death or loss to follow-up using Kaplan-Meier survival analysis. Overall survival rates were assessed using Cox proportional hazards regression analysis and proportional assumptions were verified using Schoenfeld residuals [13]. Associations between CR and other variables were evaluated using Poisson regression [14]. All statistical tests were two-sided, and $P<0.05$ was considered to indicate a statistically significant difference. Statistical analyses of the data were performed using R statistical software, version 3.1.2 [15].

\section{Results}

\subsection{Baseline Characteristics}

The age of the patients was $63 \pm 10$ years and $74 \%$ were male. The cohort characteristics are summarized in Table 1. Most (88\%) of the patients were cirrhotic (Child-Pugh A 62\% and Child-Pugh B 38\%). The etiology of the liver disease was hepatitis $C$ virus in 26/52 (50\%), hepatitis B virus in 8/52 (15\%), alcohol in 10/52 (19\%), and nonalcoholic steatohepatitis in 3/52 (6\%). According to the BCLC staging system, half of the patients were BCLC A and half were BCLC B. Most patients had one nodule (66\%). The average size of the largest nodule was $43 \pm 22 \mathrm{~mm}$ and the median of the sum of the largest diameters of all nodules was $68 \pm 37 \mathrm{~mm}$. The median alpha-fetoprotein (AFP) value was $25 \mathrm{ng} / \mathrm{ml}$ (1 - 60,500 ng/ml). No patient had extra-hepatic metastasis or macroscopic vascular invasion.

\subsection{Evaluation of Tumor Response after TACE}

Based on mRECIST criteria for assessment of tumor response, 12 patients reached CR (23\%), 19 PR (37\%), four SD (8\%) and 17 PD (32\%) (Figure 1). The relative risk of non-CR is shown in Table 2. Diameter of the largest HCC $\leq 58 \mathrm{~mm}$ and BCLC A were associated with a lower risk of non-CR $(P<0.001$ and $P<0.05$, respectively).

\subsection{Survival after the First TACE Procedure in Patients with HCC}

One-year survival after the first TACE procedure was 74\% (95\% confidence interval (CI) 63\% - 87\%) (Figure 2). Sixteen of the 52 patients had died after a mean follow-up of 404 days (95 - 526 days). The causes of death were tumor progression in 11 and liver failure in five patients.

Table 2. Relative risk of incomplete response according to simple Poisson regression.

\begin{tabular}{ccc}
\hline Variable & RR (95\% CI) & P-value \\
Male & $1.0(0.71-1.41)$ & 1.0 \\
Age $>$ 65 years old & $0.93(0.69-1.25)$ & 0.61 \\
BCLC B & $1.5(1.09-2.07)$ & 0.014 \\
Largest HCC size $>58 \mathrm{~mm}$ & $1.46(1.18-1.81)$ & $1.4(1.08-1.81)$ \\
Sum of HCCs $>74 \mathrm{~mm}$ & $1.35(0.99-1.85)$ \\
AFP $>25 \mathrm{ng} / \mathrm{ml}$ & 0.01 & 0.07 \\
\hline
\end{tabular}




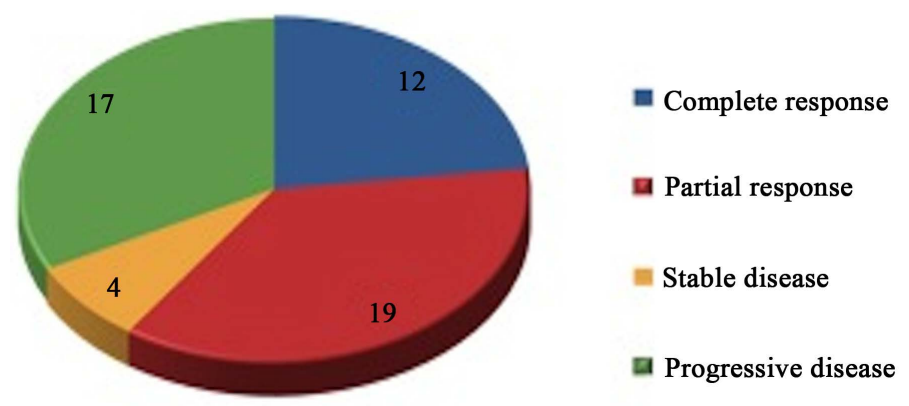

Figure 1. Initial tumor response according to mRECIST criteria.

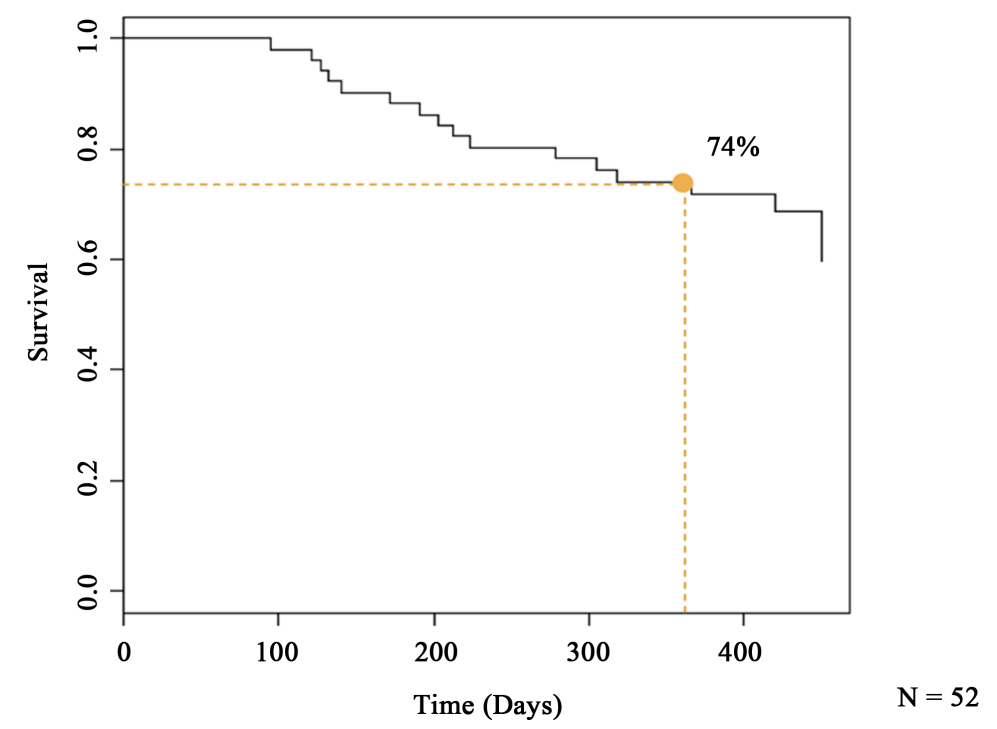

Figure 2. Overall survival.

According to univariable Cox regression analysis of pre-TACE variables, the variables associated with longer survival were closely related to tumor burden (tumor size, sum of HCCs, AFP level and BCLC stage) (Table 3). BCLC B [relative risk (RR) 6.78, 95\% CI 1.51 - 30.4, $P=0.01$ ] and AFP > 25 ng/ml (RR 3.61, 95\%CI 1.01 $12.91, P=0.04$ ) were independently associated with poor prognosis according to multivariate Cox regression analysis. Survival rates were 95\% and 56\% in the BCLC A and B groups, respectively (Figure 3).

According to mRECIST criteria, presence of CR was the best predictor of 1-year survival; the survival rate for patients with CR was $100 \%$, compared with $64 \%$ for patients with PR, SD or PD $(P=0.02)$ (Figure 4). However, only 12 patients (23\%) in the current cohort achieved CR.

Data on adverse events were not collected for this study

\section{Discussion}

TACE-DEB is an effective therapeutic option for patients with non-resectable HCC, resulting in good tumor response rates and high overall survival during 1-year of follow-up. The results of this study suggest that assessing initial tumor treatment response by mRECIST criteria, together with other tumor features such as AFP and tumor size, provide good survival predictors in patients with HCC undergoing TACE-DEB.

TACE-DEB is a drug delivery system designed to deliver a higher and more sustained release of chemoembolization drug directly into the tumor, with the intention of maximizing the drug's effectiveness. Recent evidence has established the efficacy and safety of TACE-DEB for the treatment of HCC patients. Varela et al. showed CR rates of $0 \%$ and $26 \%$, PR rates of $44 \%$ and $40 \%$, and SD rates of $26 \%$ and $4 \%$, according to mRECIST and EASL response criteria, respectively. The amended EASL response criteria provide an accepted method for assessing tumor necrosis following loco-regional therapies [7]. 


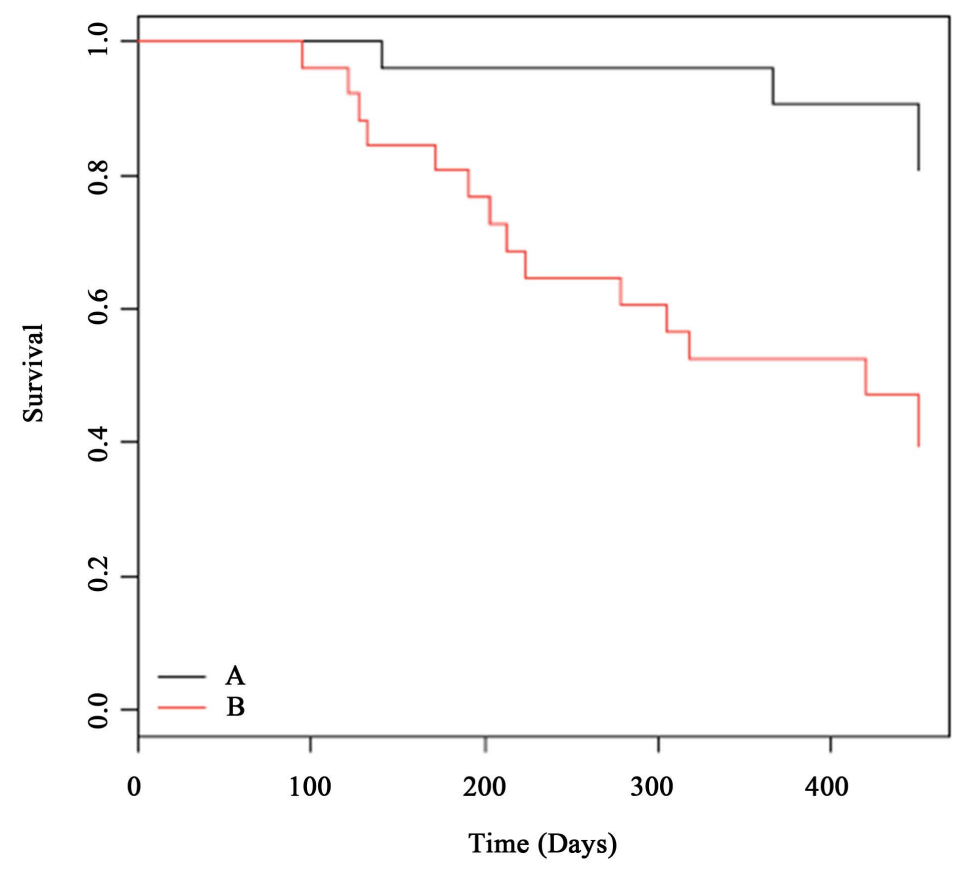

Figure 3. Survival rate according to BCLC stage.
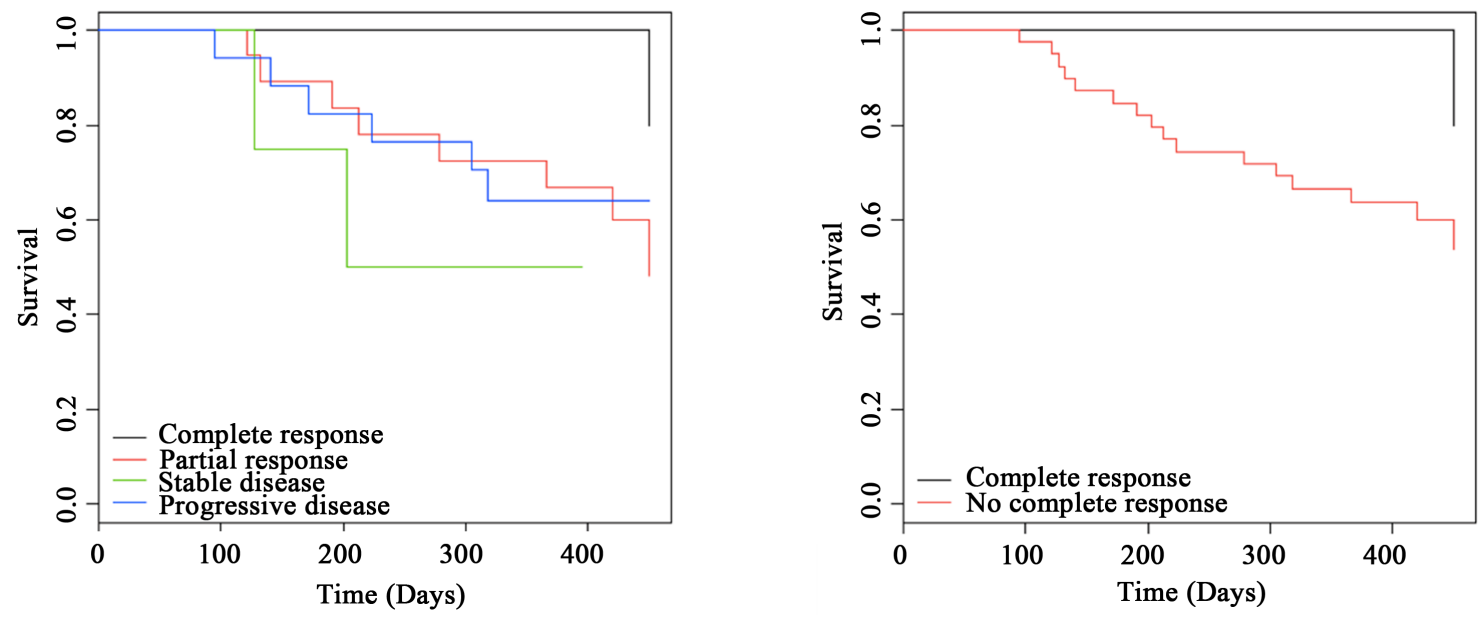

Figure 4. Survival rate according to mRECIST criteria.

Table 3. Univariable analyses.

\begin{tabular}{ccc}
\hline Variable & RR (95\% CI) & $P$-value \\
\hline Age $>$ 65 years & $1.41(0.53-3.7)$ & 0.49 \\
Male & $1.68(0.48-5.85)$ & 0.41 \\
Child-Pugh B & $2.05(0.79-5.33)$ & 0.14 \\
Number of nodules $>1$ & $0.84(0.32-2.21)$ & $<.73$ \\
Largest HCC size $>$ 58 mm & $6.12(2.26-16.57)$ & $<0.001$ \\
Sum of HCCs $>$ 74 mm & $8.14(2.81-23.6)$ & 0.001 \\
AFP $>$ 25 ng/ml & $3.81(1.24-11.71)$ & 0.001 \\
BCLC B & $6.05(1.73-21.08)$ & 0.05 \\
\hline
\end{tabular}

Size of main tumor, sum of HCCs, AFP and tumor stage were associated with poor prognosis. 
There is also increasing evidence to suggest that TACE-DEB can achieve the same or better tumor response compared with conventional TACE. PRECISION V was the first international, multicenter, randomized study designed to evaluate the efficacy of TACE-DEB compared with conventional TACE [3]. The authors demonstrated CRs in $27 \%$ vs. $22 \%$ of patients, PRs in $25 \%$ vs. $21 \%$, SD in $12 \%$ vs. $8 \%$, and PD in $32 \%$ vs. $41 \%$ in the TACE-DEB and conventional TACE groups, respectively. However, despite an overall trend favoring TACEDEB over conventional TACE, significant superiority in objective response rates was only observed in subgroups of patients with more advanced disease.

Song et al. performed a case-control study to evaluate the tumor response in 40 HCC patients treated with TACE-DEB or conventional TACE. The CR and PR rates were $55 \%$ and $26.6 \%$ in the TACE-DEB group, and $23.1 \%$ and $26.3 \%$ in the conventional TACE group, respectively $(P<0.001)$. The response rates in the TACEDEB group were higher than those reported in previous studies, but this could be attributable to the retrospective study design and selection bias [4].

The current study found a CR rate of $23 \%$, PR rate of $37 \%$, SD rate of $8 \%$, and PD rate of $32 \%$ after the first procedure. These results are consistent with previous data for TACE-DEB, and show equivalent or superior efficacy compared with conventional TACE based on currently available published studies.

However, there are conflicting results regarding the relative effects of TACE-DEB and conventional TACE on overall survival [8] [9]. Huang et al. performed a meta-analysis including seven studies and 700 patients and demonstrated that 1-year and 2-year survival rates were better after TACE-DEB compared with conventional TACE [8]. In contrast, a recent systematic review demonstrated that, although TACE-DEB was associated with better tumor response and potentially fewer adverse events, it did not confer a greater survival benefit compared with conventional TACE [9].

The clinical selection of patients may influence the survival rate. For example, following a strict patient selection procedure (preserved liver function, absence of symptoms, extra-hepatic spread or vascular invasion), Burrel et al. achieved a 1-year survival of $89.9 \%$, with a median overall survival of 48.6 months (95\%CI: 36.9 61.2) after treatment with TACE-DEB [10]. In contrast, a prospective analysis by Skowasch et al. [11] presented cumulative survival rates at 1 and 2 - 4 years of $66.7 \%$ and $45.7 \%$, respectively. However, these authors also included patients classified as BCLC C for TACE-DEB, so long as the portal vein thrombosis did not affect the main portal vein.

The current study confirmed promising survival rates with TACE-DEB, with a 1-year survival of $74 \%$ during a mean follow-up of 404 days after the first procedure, based on inclusion criteria of non-resectable HCC, liverconfined disease, good performance status ( 0 - 1 ) and adequate liver function (Child-Pugh A/B).

In 2004, the BCLC group demonstrated that evaluation of initial CR to percutaneous ablation was associated with improved survival rates and should be considered as a relevant therapeutic target [12]. More recently, Gilmore et al. compared the use of EASL, mRECIST, and RECIST criteria in patients undergoing TACE, and concluded that EASL and mRECIST overall response assessments were associated with longer survival, and should be used in preference to RECIST criteria [2].

We found that reduction in tumor size after the first TACE-DEB procedure was associated with better survival, with 100\% 1-year survival in patients with CR. We also demonstrated associations between survival and variables related to tumor extension (tumor size, sum of HCCs) and BCLC stage before TACEDEB.

This study was limited by its retrospective design, relatively small sample size, and the fact that tumor treatment response was assessed by a single radiologist.

\section{Conclusion}

In conclusion, the present study demonstrated that BCLC A and the presence of CR based on mRECIST criteria were good predictors of 1-year survival in patients with HCC undergoing TACE-DEB. Further studies are needed to evaluate other survival predictors and to determine if tumor response assessment also predicts long-term survival.

\section{Disclosure Statement}

All authors declare that they have no conflicts of interest. 


\section{References}

[1] Kang, H., Lee, H.Y., Lee, K.S. and Kim, J.-H. (2012) Imaging-Based Tumor Treatment Response Evaluation: Review of Conventional, New, and Emerging Concepts. Korean Journal of Radiology, 13, 371-390. http://dx.doi.org/10.3348/kjr.2012.13.4.371

[2] Gillmore, R., Stuart, S., Kirkwood, A., Hameeduddin, A., Woodward, N., Burroughs, A.K. and Meyer, T. (2011) EASL and mRECIST Responses Are Independent Prognostic Factors for Survival in Hepatocellular Cancer Patients Treated with Transarterial Embolization. Journal of Hepatology, 55, 1309-1316. http://dx.doi.org/10.1016/j.jhep.2011.03.007

[3] Bruix, J. and Sherman, M. (2011) Management of Hepatocellular Carcinoma: An Update. Hepatology, 53, $1020-1022$. http://dx.doi.org/10.1002/hep.24199

[4] Bruix, J., Sherman, M., Llovet, J.M., Beaugrand, M., Lencioni, R., Burroughs, A.K., Christensen, E., Pagliaro, L., Colombo, M. and Rodés, J. (2001) Clinical Management of Hepatocellular Carcinoma. Conclusions of the Barcelona2000 EASL Conference. European Association for the Study of the Liver. Journal of Hepatology, 35, 421-430. http://dx.doi.org/10.1016/S0168-8278(01)00130-1

[5] Lammer, J., Malagari, K., Vogl, T., Pilleul, F., Denys, A., Watkinson, A., Pitton, M., Sergent, G., Pfammatter, T., Terraz, S., Benhamou, Y., Avajon, Y., Gruenberger, T., Pomoni, M., Langenberger, H., Schuchmann, M., Dumortier, J., Mueller, C., Chevallier, P. and Lencioni, R. (2010) Prospective Randomized Study of Doxorubicin-Eluting-Bead Embolization in the Treatment of Hepatocellular Carcinoma: Results of the PRECISION V Study. CardioVascular and Interventional Radiology, 33, 41-52. http://dx.doi.org/10.1007/s00270-009-9711-7

[6] Song, M.J., Chun, H.J., Song, D.S., Kim, H.Y., Yoo, S.H., Park, C.-H., Bae, S.H., Choi, J.Y., Chang, U.I., Yang, J.M., Lee, H.G. and Yoon, S.K. (2012) Comparative Study between Doxorubicin-Eluting Beads and Conventional Transarterial Chemoembolization for Treatment of Hepatocellular Carcinoma. Journal of Hepatology, 57, 1244-1250. http://dx.doi.org/10.1016/j.jhep.2012.07.017

[7] Lencioni, R. and Llovet, J.M. (2010) Modified RECIST (mRECIST) Assessment for Hepatocellular Carcinoma. Seminars in Liver Disease, 30, 52-60. http://dx.doi.org/10.1055/s-0030-1247132

[8] Huang, K., Zhou, Q., Wang, R., Cheng, D. and Ma, Y. (2014) Doxorubicin-Eluting Beads versus Conventional Transarterial Chemoembolization for the Treatment of Hepatocellular Carcinoma. Journal of Gastroenterology and Hepatology, 29, 920-925. http://dx.doi.org/10.1111/jgh.12439

[9] Xie, Z.-B., Wang, X.-B., Peng, Y.-C., Zhu, S.-L., Ma, L., Xiang, B.-D., Gong, W.-F., Chen, J., You, X.-M., Jiang, J.-H., Li, L.-Q. and Zhong, J.-H. (2014) Systematic Review Comparing the Safety and Efficacy of Conventional and Drug-Eluting-Bead Transarterial Chemoembolization for Inoperable Hepatocellular Carcinoma. Hepatology Research, 45, 190-200.

[10] Burrel, M., Reig, M., Forner, A., Barrufet, M., de Lope, C.R., Tremosini, S., Ayuso, C., Llovet, J.M., Real, M.I. and Bruix, J. (2012) Survival of Patients with Hepatocellular Carcinoma Treated by Transarterial Chemoembolisation (TACE) Using Drug Eluting Beads. Implications for Clinical Practice and Trial Design. Journal of Hepatology, 56, 1330-1335. http://dx.doi.org/10.1016/j.jhep.2012.01.008

[11] Skowasch, M., Schneider, J., Otto, G., Weinmann, A., Woerns, M.A., Dueber, C. and Pitton, M.B. (2012) Midterm Follow-Up after DC-BEAD ${ }^{\mathrm{TM}}$-TACE of Hepatocellular Carcinoma (HCC). European Journal of Radiology, 81, 38573861. http://dx.doi.org/10.1016/j.ejrad.2012.07.002

[12] Sala, M., Llovet, J.M., Vilana, R., Bianchi, L., Solé, M., Ayuso, C., Brú, C. and Bruix, J. (2004) Initial Response to Percutaneous Ablation Predicts Survival in Patients with Hepatocellular Carcinoma. Hepatology, 40, 1352-1360. http://dx.doi.org/10.1002/hep.20465

[13] Schoenfeld, D. (1982) Partial Residuals for the Proportional Hazards Regression Model. Biometrika, 69, $239-241$. http://dx.doi.org/10.1093/biomet/69.1.239

[14] Zou, G. (2004) A Modified Poisson Regression Approach to Prospective Studies with Binary Data. American Journal of Epidemiology, 159, 702-706. http://dx.doi.org/10.1093/aje/kwh090

[15] R Development Core Team (2014) R: A Language and Environment for Statistical Computing. R Foundation for Statistical Computing, Vienna, Austria. R Foundation for Statistical Computing, Vienna. 


\section{List of Abbreviations}

HCC

TACE

DEB

TACE-DEB

RECIST

mRECIST

EASL

AASLD

WHO

ICESP

CT

MRI

CR

PR

PD

SD
Hepatocellular carcinoma

Transarterial chemoembolization

Drug-eluting beads

Chemoembolization with drug-eluting beads

Response evaluation criteria in solid tumors

modified RECIST

European Association for the Study of the Liver

American Association for the Study of Liver Diseases

World Health Organization

Instituto do Câncer do Estado de São Paulo

Computed tomography

Magnetic resonance imaging

Complete response

Partial response

Disease progression

Stable disease 